\title{
ALGORITMO PARA COMPLETAR SERIES DE VALORES DE LA FRECUENCIA CRÍTICA DE LA REGIÓN F DE LA IONÓSFERA SOBRE CONCEPCIÓN, CHILE
}

\author{
M.A. Arriagada ${ }^{1} \&$ A. J. Foppiano ${ }^{2}$
}

\begin{abstract}
Se usa la serie incompleta de valores horarios de la foF2 sobre Concepción $\left(36.8^{\circ} \mathrm{S} ; 73.0^{\circ} \mathrm{O}\right)$, observados en el intervalo 1958 - 1989, para determinar inicialmente una serie completa de valores horarios de foF2 que pueden considerarse representativos de condiciones geomagnéticas quietas, pero que toma en cuenta las dependencias de la foF 2 con la estación del año y con el nivel de actividad solar. Luego se propone un algoritmo para calcular valores horarios faltantes en el intervalo, en que sí se toma en cuenta el nivel de actividad geomagnética observado en las horas correspondientes, usando un índice de actividad geomagnética ponderado. Como evidentemente no se puede determinar la bondad del algoritmo propuesto para las horas en que no ha sido posible medir la foF2, se compara los valores horarios calculados con los observados, para días en que no hay ningún valor faltante, y que se consideran representativos de diferentes estaciones del año y niveles de actividad solar y geomagnética. Se encuentra que las diferencias no son significativas a excepción de días de equinoccio y verano en condiciones geomagnéticas muy perturbadas.
\end{abstract}

Palabras claves: Frecuencia crítica; Región F; Ionósfera.

ALGORITHM TO FILL IN IONOSPHERIC F-REGION CRITICAL FREQUENCY SERIES FOR CONCEPCIÓN, CHILE - A complete series of hourly values of foF 2 considered representative of geomagnetic quiet conditions is determined using the incomplete series of observed hourly values at Concepción $\left(36.8^{\circ} \mathrm{S} ; 73.0^{\circ} \mathrm{W}\right)$ in the 1 March $1958-1 \mathrm{July} 1989$ interval. This series takes into account known seasonal and solar activity level dependencies. Then a simple algorithm is proposed to calculate the missing hourly values of the original series, which takes into account the geomagnetic activity level for the corresponding hours, in terms of a weighted geomagnetic activity index. Since there is no way to assert the goodness of the proposed algorithm when there is no observed foF 2 values, calculated and observed values are compared for a few days for which they do exist. Days considered representative of different seasons and levels of solar and geomagnetic activity are used. It is found that differences between calculated and observed values are not significant except for equinox and summer conditions at high geomagnetic activity level.

Key words: Critical frequency; F-region; Ionosphere.

\author{
'Departamento de Física, Universidad del Bío-Bío, Casilla 5-C Concepción, \\ Teléfono $+56-41-261219$ \\ Fax +56-41-320068 \\ marriga@ubiobio.cl \\ ${ }^{2}$ Departamento de Física de la Atmósfera y del Océano, Universidad de Concepción, \\ Casilla 4009, Concepción, Chile, \\ Teléfono $+56-41-203083$ \\ Fax +56-41-220104 \\ foppiano@udec.cl
}




\section{INTRODUCCIÓN}

En el análisis de la larga serie de valores de la frecuen crítica de la región $\mathrm{F}$, foF 2 , sobre Concepción $\left(36.8^{\circ} \mathrm{S} ; 7\right.$ ? O), invariablemente se presenta el problema de comple la serie por la falta de registros, debidos a fallas en equipos, a errores humanos, o a condiciones ionosféri que impiden la obtención de valores numéricos. $\mathrm{P}$ completar la serie se puede hacer uso de diver: procedimientos de interpolación, pudiendo elegirse el $\mathrm{r}$ apropiado de entre ellos, dependiendo fundamentalme del número de valores horarios faltantes en relación con conocidas variaciones regulares, diurna, estacional y cor nivel de actividad solar. Estos procedimientos no ton normalmente en cuenta otras variaciones, de carác episódico, como las que se observan en los regist ionosféricos, por ejemplo en asociación con la ocurren de llamaradas solares y de eyecciones de masa de la cors solar.

El objetivo del presente trabajo es proponer un algorit que permita determinar los valores horarios faltantes $\mathrm{d} t$ foF2, para cualquier hora del día en el intervalo 1958 - 1989, el que se fundamenta en la determinación empírica de las dependencias de la foF 2 con los niveles de actividades solar y geomagnética. Se estima que este procedimiento da una mejor cuenta de las variaciones regulares y de las episódicas, además de permitir la completación de valores faltantes, incluso para varios meses consecutivos. Aunque el algoritmo es obviamente válido solo para Concepción durante el intervalo considerado, se podría sugerir su aplicación a otras localidades que cuenten con series de valores comparables.

\section{METODOLOGÍA}

La metodología usada sigue estrechamente la propuesta por Wrenn et al. (1987) y Rodger et al. (1989) para el estudio del efecto de tormentas geomagnéticas en la región $\mathrm{F}$.

En primer lugar se determinó el índice ponderado de actividad geomagnética acumulada, ap (0.75), definido inicialmente por Wrenn (1987), para cada intervalo trihorario desde las 05:00 horas Tiempo Universal (TU) del 1 de marzo de 1958 (00:00 horas de Tiempo Local (TL) para el huso horario de $75^{\circ} \mathrm{O}$ ) hasta las 04:00 horas TU del 1 de julio de 1989 (23:00 horas TL).

Luego se agruparon para cada hora los valores horarios de la foF2 de todos los días de un mes determinado, correspondientes al intervalo 1 de noviembre de 1968 a 30 de noviembre de 1979, para los cuales el nivel de actividad geomagnética es bajo (índice trihorario $a p(0.75)<6$ ) y el nivel de actividad solar (flujo solar del día en $10.7 \mathrm{~cm}, \mathrm{~F} 10.7$ ) está en uno de cuatro rangos: 1 (50 d F10.7 < 100); 2 (100 d F10.7 < 150); 3 (150 d F10.7 < 200) y 4 (200 d F10.7 < 250). Este intervalo se eligió inicialmente para que los resultados del presente análisis sean directamente comparables con los resultados de Wreen et al., (1987). Seguidamente se calcularon valores medios mensuales para

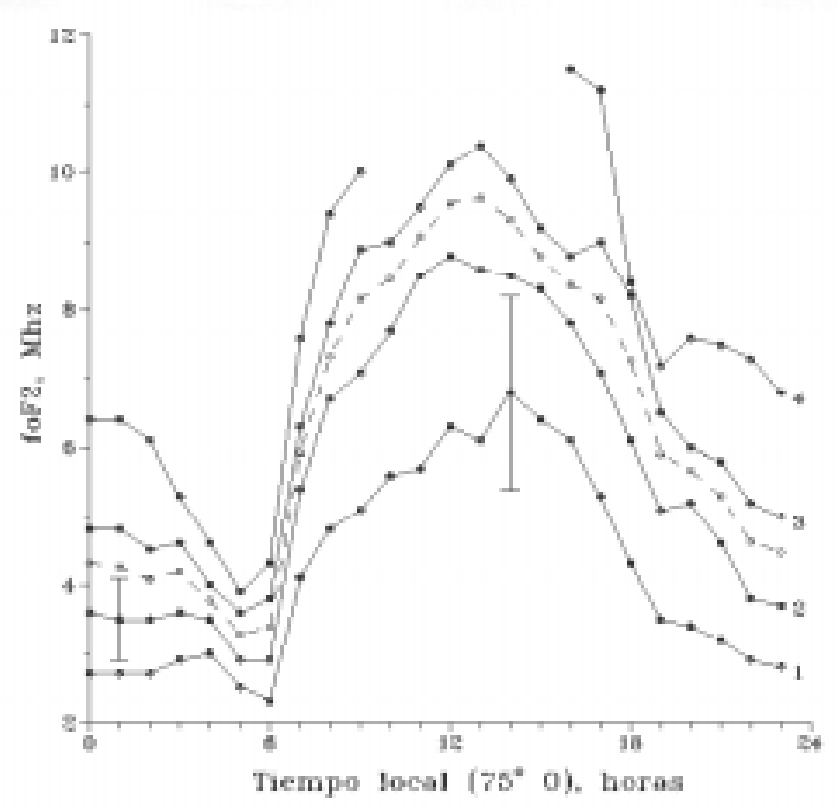

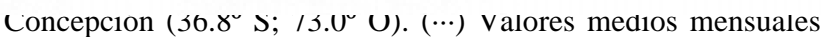
calculados usando los valores horarios de todos los días de los meses de agosto del intervalo 1 de noviembre de 1968 al 30 de noviembre de 1979, para los cuales el nivel de actividad geomagnética es bajo (índice trihorario $a p(0.75)<6$ ) y el nivel de actividad solar (flujo solar del día en $10.7 \mathrm{~cm}$ ) está en uno de cuatro rangos: 1 (50 d F10.7 < 100); 2 (100 d F10.7 < 150); 3 (150 d F10.7 < 200) y 4 (200 d F10.7 < 250). Para algunas horas las dos condiciones no se cumplen simultáneamente en el rango 4. (ooo) Valores mensuales representativos, foF 2 , para agosto de 1969 (F10.7 medio mensual 146.1) calculados por interpolación lineal entre los correspondientes a los niveles de actividad solar 2 y 3 (118.26 a 121.13, y 164.26 a 167.61 , respectivamente, dependiendo de la hora del día). Las barras de variabilidad ( \pm una desviación estándar) indican las variabilidades máximas encontradas entre las 00 y 06, y las 06 y 18 horas, respectivamente. A otras horas las variabilidades son similares.

Figure 1 - Ionospheric F-region critical frequency observed over Concepción $\left(36.8^{\circ} \mathrm{S} ; 73.0^{\circ} \mathrm{W}\right)$. ( ...) Monthly mean values determined using hourly values for all days from the 1 November 1968 - 30 November 1979 interval, corresponding geomagnetic activity low level (three-hourly index ap $(0.75)<6)$ and four solar activity levels (daily $10.7 \mathrm{~cm}$ solar flux): 1 (50 d F10.7 < 100); 2 (100 d F10.7 < 150); 3 (150 d F10.7 < 200) y 4 (200 d F10.7 < 250). Both conditions are not simultaneously met for some hours at 4 level. (ooo) Monthly values, foF $2_{m,}$, for August 1969 determined by linear interpolation between corresponding values for solar activity levels 2 and 3 (118.26 to 121.13, and 164.26 to167.61, respectively, depending on time-of-day). Variability bars ( \pm one standard deviation) indicate maxima variabilities found between 00 and 06 , and 06 to 18 hours, respectively. Variabilities for other times-of-day are similar.

cada hora, usando los valores horarios de todos los días así agrupados para cada mes. En la Fig. 1 se muestran estos valores medios mensuales para el caso del mes de agosto, los que ilustran las dependencias empíricas de la foF 2 con el nivel de actividad solar para el mes en cuestión. Se hace 


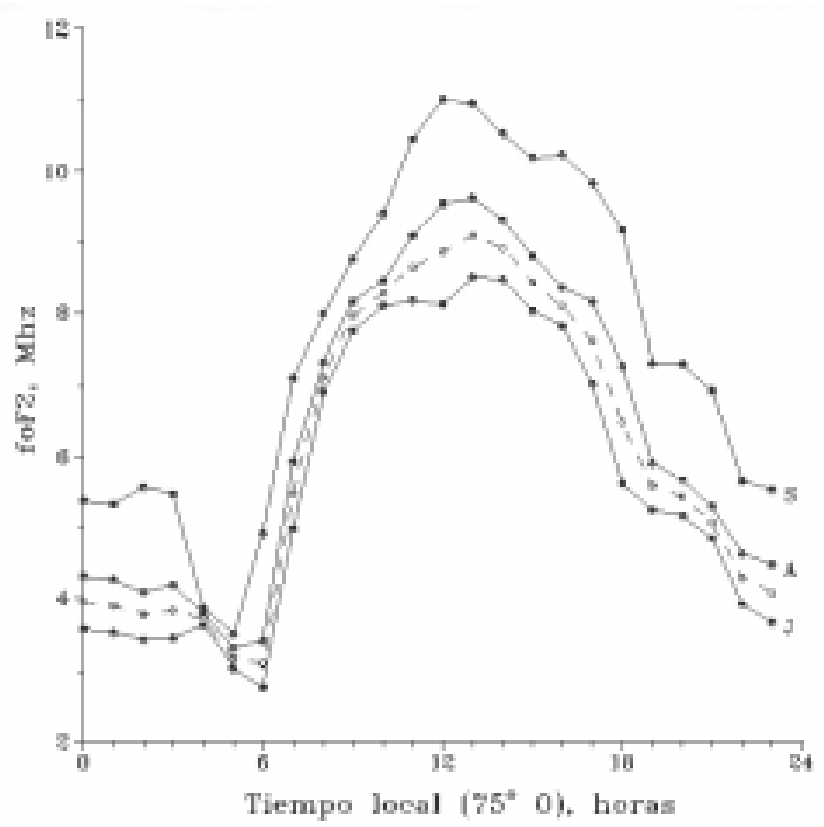

bajo (índice trihorario ap $(\hat{0.75)}<6)$. ( ...) Valorěs mensuales representativos, foF $2_{\mathrm{mr}}$, para julio (J), agosto (A) y septiembre (S) de 1969, calculados como los que se muestran en la Fig. 1 para el caso de agosto de 1969, y asignados al día mediano del mes correspondiente. (ooo) Valores horarios representativos, foF 2 dr, para el 1 de agosto de 1969 calculados por interpolación lineal entre los correspondientes a los días medianos del julio y agosto.

Figure 2 - Ionospheric F-region critical frequency observed over Concepción $\left(36.8^{\circ} \mathrm{S} ; 73.0^{\circ} \mathrm{W}\right)$ for low level geomagnetic activity (three-hourly index ap $(0.75)<6)$. (...) Monthly values, foF $2_{m r}$, for July (J), August (A) and September (S) 1969, determined as those shown in Fig. 1 for August 1969 and assigned to the median day of corresponding month. (ooo) Hourly values, foF $2_{d r}$, for 1 August 1969 determined using linear interpolation between those corresponding to July and August median days.

notar que, para algunas horas, la condición nivel de actividad geomágnética baja no se cumple en el rango 4 de nivel de actividad solar.

A continuación se calculó el valor medio mensual del F10.7 para cada mes del intervalo considerado. Se determinó enseguida, para cada hora del día, valores de la foF2 representativos para cada mes del intervalo, foF ${ }_{\mathrm{mr}}$, por interpolación lineal entre los valores de la foF2 correspondientes a dos niveles de actividad solar sucesivos del mes considerado. En la Fig. 1 se muestran los valores representativos así calculados para agosto de 1969. El valor medio del F10.7 para agosto de 1969 es 146.1, que está entre los valores del F10.7 correspondientes a agosto nivel 2 (118.26 a 121.13, dependiendo de la hora del día) y a agosto nivel 3 (164.26 a 167.61).

El siguiente paso fue asignar esos valores representativos de cada mes al día mediano del mes, por ejemplo al día 16 en los meses de 31 días, o al "día 15.5" para los meses de 30 días. En la Fig. 2 se muestran los valores foF ${ }_{\mathrm{mr}}$

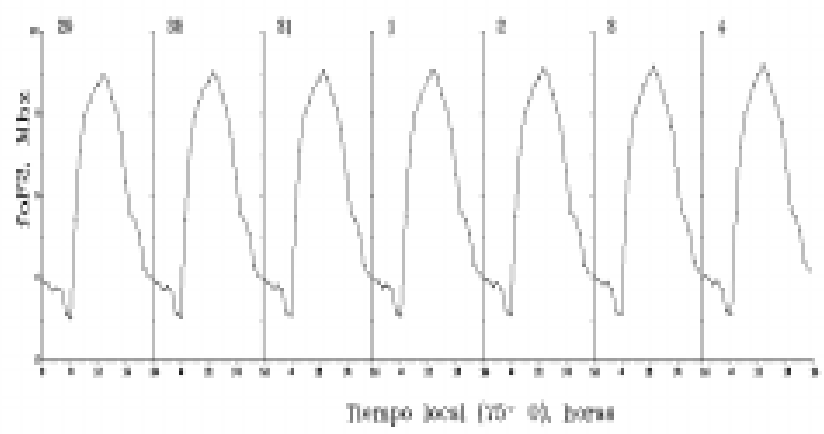

iigura 3 - Frecuencia crítica de la región $\mathrm{F}$ de la ionósfera sobre Joncepción $\left(36.8^{\circ} \mathrm{S} ; 73.0^{\circ} \mathrm{O}\right)$ para nivel de actividad geomagnética ajo (índice trihorario $a p(0.75)<6$ ). Valores horarios epresentativos, foF $2_{\mathrm{dr}}$, para los días 29 de julio al 4 de agosto de 969, calculados como los que se muestran en la Fig. 2 para el aso del 1 de agosto de 1969.

igure 3 - Ionospheric F-region critical frequency observed over Ioncepción $\left(36.8^{\circ} \mathrm{S} ; 73.0^{\circ} \mathrm{W}\right)$ for low level geomagnetic activity three-hourly index ap $(0.75)<6$ ). Hourly values, foF ${ }_{d r}$, for 29 uly to 4 August 1969, determined as those shown in Fig. 2 for the August 1969 case.

correspondientes a julio, agosto y septiembre de 1969 . Luego se determinaron valores horarios, foF ${ }_{\mathrm{dr}}$, para todos los días entre los días medianos de dos meses sucesivos, por interpolación lineal entre los valores foF ${ }_{\mathrm{mr}}$ asignados a esos meses. En la Fig. 2 se muestra estos valores foF ${ }_{\mathrm{dr}}$ calculados para el día 1 de agosto de 1969. En la Fig. 3 se muestran los valores foF 2 dr para los días 29 de julio al 4 de agosto de 1969, los que ilustran los pequeños cambios debidos a las dependencias con la estación del año y con el nivel de actividad solar. De la misma manera se determinó valores foF ${ }_{\mathrm{dr}}$ para cada hora de cada día del intervalo considerado, que se pueden considerar como típicos de condiciones geomagnéticas quietas, para el nivel de actividad solar que le corresponde, pues las dependencias empíricas con el nivel de actividad solar ya han sido incluidas.

Seguidamente se determinó la dependencia de la foF2 con el nivel de actividad geomagnética. Para ello se calculó el cuociente, q, entre el valor de la foF2 observada y la foF2 representativa de condiciones geomagnéticas quietas, $\mathrm{foF}_{\mathrm{dr}}$, para cada hora de todo el intervalo inicialmente elegido (1 de noviembre de 1968 a 30 de noviembre de 1979), y se agrupó estos cuocientes de acuerdo con la hora del día, mes del año y cuatro rangos de actividad geomagnética seleccionados por el valor del $a p(0.75)$ (muy quieto: $0 \mathrm{~d}$ $a p(0.75)<6$; quieto: $6 \mathrm{~d}$ ap $(0.75)<18$; perturbado: $18 \mathrm{~d}$ $a p(0.75)<30$; y muy perturbado: $30 \mathrm{~d} a p(0.75))$. En la Fig. 4a se muestran los valores de q así obtenidos para el mes de agosto, lo que ilustra las dependencias empíricas de la foF2 con el nivel de actividad geomagnética para el mes en cuestión. Se advierte que las dependencias para los distintos meses de una misma estación del año son muy similares, por lo que se calcula valores medios de q para equinoccio (marzo, abril, septiembre y octubre), invierno (mayo, junio, julio y agosto), y verano (noviembre, diciembre, enero y 

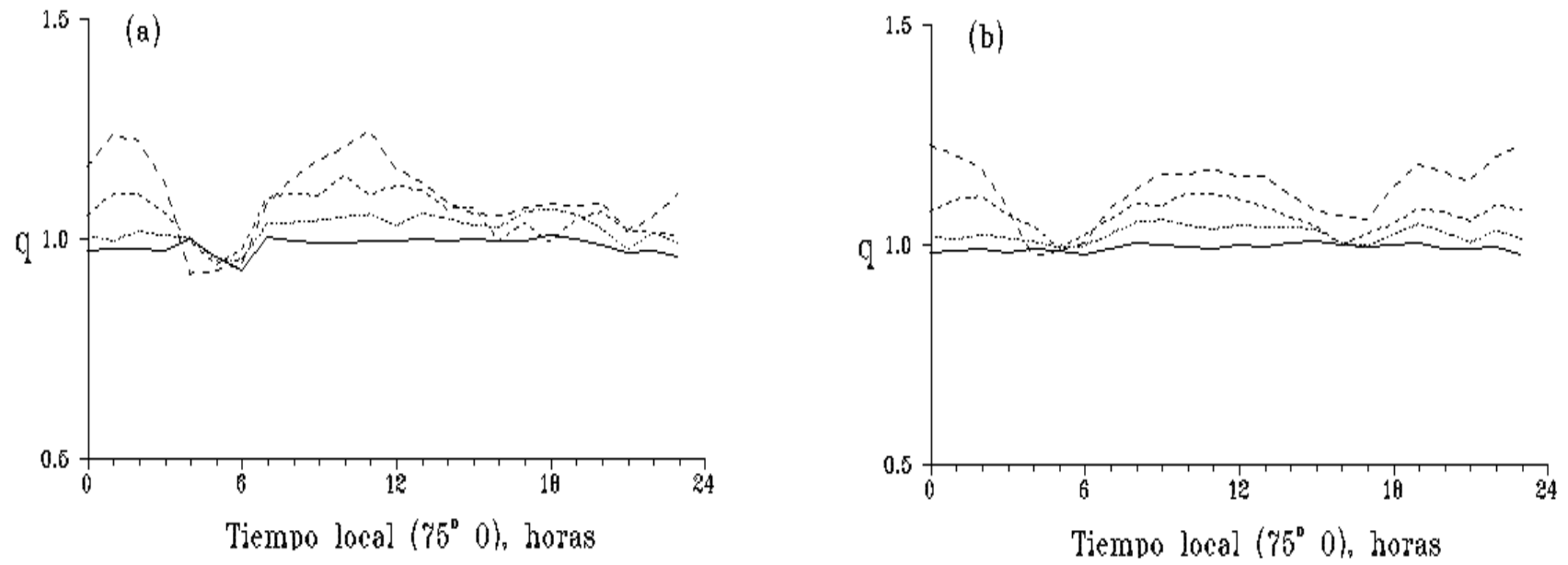

noviembre de 1968 al 30 de noviembre de 1979 (ver texto). --- Muy quieto (0 d ap (0.75) < 6); LLL Quieto $(6 \mathrm{~d}$ ap $(0.75)<18)$; Perturbado (18 d ap (0.75) < 30); y - - - Muy perturbado (30 d ap (0.75)). (a) Agosto. (b) Invierno.

Figure 4 - Ratio between ionospheric F-region critical frequency observed over Concepción $\left(36.8^{\circ} S ; 73.0^{\circ} O\right)$ and critical frequency determined for low geomagnetic activity level (three-hourly index ap $(0.75)<6)$ corresponding to 1 November 1968 - 30 November 1979 (see text). --- very quiet $(0 \mathrm{~d}$ ap $(0.75)<6)$; LLL quiet $(6 \mathrm{~d}$ ap $(0.75)<18)$; — perturbed $(18 \mathrm{~d}$ ap $(0.75)<30) ;$ and - - - very perturbed (30 d ap (0.75). (a) August. (b) Winter.

INVIERNO

Muy
TL quieto
Quieto Perturbado Perturbado

$\begin{array}{rllll}0 & 0.983 & 1.019 & 1.074 & 1.226 \\ 1 & 0.988 & 1.012 & 1.104 & 1.203 \\ 2 & 0.992 & 1.024 & 1.110 & 1.173 \\ 3 & 0.982 & 1.014 & 1.067 & 1.089 \\ 4 & 0.992 & 1.008 & 1.043 & 0.974 \\ 5 & 0.987 & 0.993 & 0.995 & 0.987 \\ 6 & 0.978 & 0.998 & 1.021 & 1.006 \\ 7 & 0.990 & 1.023 & 1.059 & 1.082 \\ 8 & 1.004 & 1.052 & 1.094 & 1.123 \\ 9 & 0.998 & 1.056 & 1.088 & 1.161 \\ 10 & 0.996 & 1.044 & 1.118 & 1.162 \\ 11 & 0.992 & 1.035 & 1.115 & 1.172 \\ 12 & 0.999 & 1.044 & 1.105 & 1.155 \\ 13 & 0.996 & 1.040 & 1.087 & 1.155 \\ 14 & 1.005 & 1.041 & 1.062 & 1.114 \\ 15 & 1.007 & 1.034 & 1.044 & 1.076 \\ 16 & 1.001 & 1.003 & 1.001 & 1.066 \\ 17 & 0.994 & 0.998 & 1.024 & 1.056 \\ 18 & 0.999 & 1.025 & 1.048 & 1.132 \\ 19 & 1.005 & 1.048 & 1.083 & 1.183 \\ 20 & 0.990 & 1.028 & 1.074 & 1.165 \\ 21 & 0.989 & 1.005 & 1.055 & 1.145 \\ 22 & 0.995 & 1.033 & 1.090 & 1.201 \\ 23 & 0.976 & 1.013 & 1.079 & 1.226\end{array}$

EQUINOCCIO

\begin{tabular}{cccc}
$\begin{array}{c}\text { Muy } \\
\text { quieto }\end{array}$ & Quieto & \multicolumn{3}{c}{ Perturbado $\begin{array}{c}\text { Muy } \\
\end{array}$} & & & \\
1.036 & 1.038 & 1.063 & 1.172 \\
1.030 & 1.023 & 1.075 & 1.169 \\
1.030 & 1.030 & 1.092 & 1.185 \\
1.037 & 1.050 & 1.086 & 1.136 \\
0.997 & 0.978 & 0.983 & 0.963 \\
0.977 & 0.976 & 1.030 & 1.012 \\
1.053 & 1.075 & 1.090 & 1.116 \\
1.042 & 1.069 & 1.083 & 1.110 \\
1.038 & 1.080 & 1.123 & 1.181 \\
1.030 & 1.064 & 1.127 & 1.153 \\
1.038 & 1.067 & 1.130 & 1.151 \\
1.027 & 1.068 & 1.133 & 1.146 \\
1.035 & 1.072 & 1.135 & 1.146 \\
1.032 & 1.074 & 1.117 & 1.132 \\
1.032 & 1.073 & 1.132 & 1.137 \\
1.030 & 1.058 & 1.109 & 1.109 \\
1.041 & 1.057 & 1.100 & 1.113 \\
1.023 & 1.039 & 1.075 & 1.127 \\
1.037 & 1.050 & 1.105 & 1.177 \\
1.053 & 1.082 & 1.143 & 1.239 \\
1.038 & 1.029 & 1.073 & 1.147 \\
1.036 & 1.009 & 1.051 & 1.129 \\
1.048 & 1.044 & 1.070 & 1.145 \\
1.037 & 1.036 & 1.063 & 1.156
\end{tabular}

VERANO

\begin{tabular}{cccc}
$\begin{array}{c}\text { Muy } \\
\text { quieto }\end{array}$ & Quieto & \multicolumn{3}{c}{$\begin{array}{c}\text { Merturbado } \\
\text { Muy }\end{array}$} \\
& & & \\
1.026 & 1.030 & 1.038 & 1.000 \\
1.031 & 1.039 & 1.046 & 1.050 \\
1.023 & 1.023 & 1.042 & 1.072 \\
1.019 & 1.008 & 1.023 & 1.032 \\
1.019 & 1.055 & 1.047 & 1.036 \\
1.021 & 1.052 & 1.028 & 1.036 \\
1.015 & 1.041 & 1.042 & 0.967 \\
1.020 & 1.011 & 0.997 & 0.981 \\
1.019 & 1.011 & 1.007 & 0.960 \\
1.007 & 1.004 & 1.008 & 0.910 \\
1.002 & 0.992 & 0.996 & 0.928 \\
1.002 & 0.996 & 1.013 & 0.942 \\
1.003 & 0.998 & 1.010 & 0.950 \\
0.998 & 0.995 & 1.006 & 0.952 \\
1.004 & 1.018 & 1.043 & 0.974 \\
1.000 & 1.007 & 1.012 & 1.016 \\
1.002 & 1.028 & 1.055 & 0.995 \\
0.999 & 1.032 & 1.059 & 1.072 \\
0.995 & 1.036 & 1.062 & 1.087 \\
0.995 & 1.029 & 1.039 & 1.104 \\
1.011 & 1.034 & 1.045 & 1.121 \\
1.019 & 1.047 & 1.076 & 1.117 \\
1.021 & 1.029 & 1.036 & 1.104 \\
1.031 & 1.028 & 1.036 & 1.074
\end{tabular}

crítica determinada para condiciones de nivel de actividad geomagnética bajo (índice trihorario ap $(0.75)<6$ ), correspondientes al intervalo 1 de noviembre de 1968 al 30 de noviembre de 1979, para distintas horas del día, estaciones del año y niveles de actividad geomagnética: Muy quieto $(0 \mathrm{~d} a p(0.75)<6)$; Quieto $(6 \mathrm{~d} a p(0.75)<18)$; Perturbado $(18 \mathrm{~d} a p(0.75)<30)$; y Muy perturbado $(30 \mathrm{~d}$ ap $(0.75)$.

Table 1 - Ratio between ionospheric F-region critical frequency observed over Concepción $\left(36.8^{\circ} S ; 73.0^{\circ} O\right)$ and critical frequency determined for low geomagnetic activity level (three-hourly index ap $(0.75)<6$ ) corresponding to 1 November $1968-30$ November 1979 , for different times-of-day, seasons and geomagnetic activity levels: very quiet $(0$ d ap $(0.75)<6)$; quiet $(6$ d ap $(0.75)<18)$; perturbed (18 $d$ ap $(0.75)<30)$; and very perturbed (30 d ap (0.75). 
febrero). La Fig. 4b ilustra las dependencias para invierno. Estos resultados son consistentes con los publicados para un intervalo equivalente por Wrenn et al. (1987) y Rodger et al. (1989), correspondientes a otro lugar del hemisferio Sur, y por Field \& Rishbeth (1997) para 53 lugares distribuidos en ambos hemisferios, incluyendo Concepción.

Así, finalmente, el valor faltante de la foF2 para cualquier hora de un día determinado se puede calcular mediante la expresión:

$$
\text { foF } 2=q \text { foF } 2_{d r}
$$
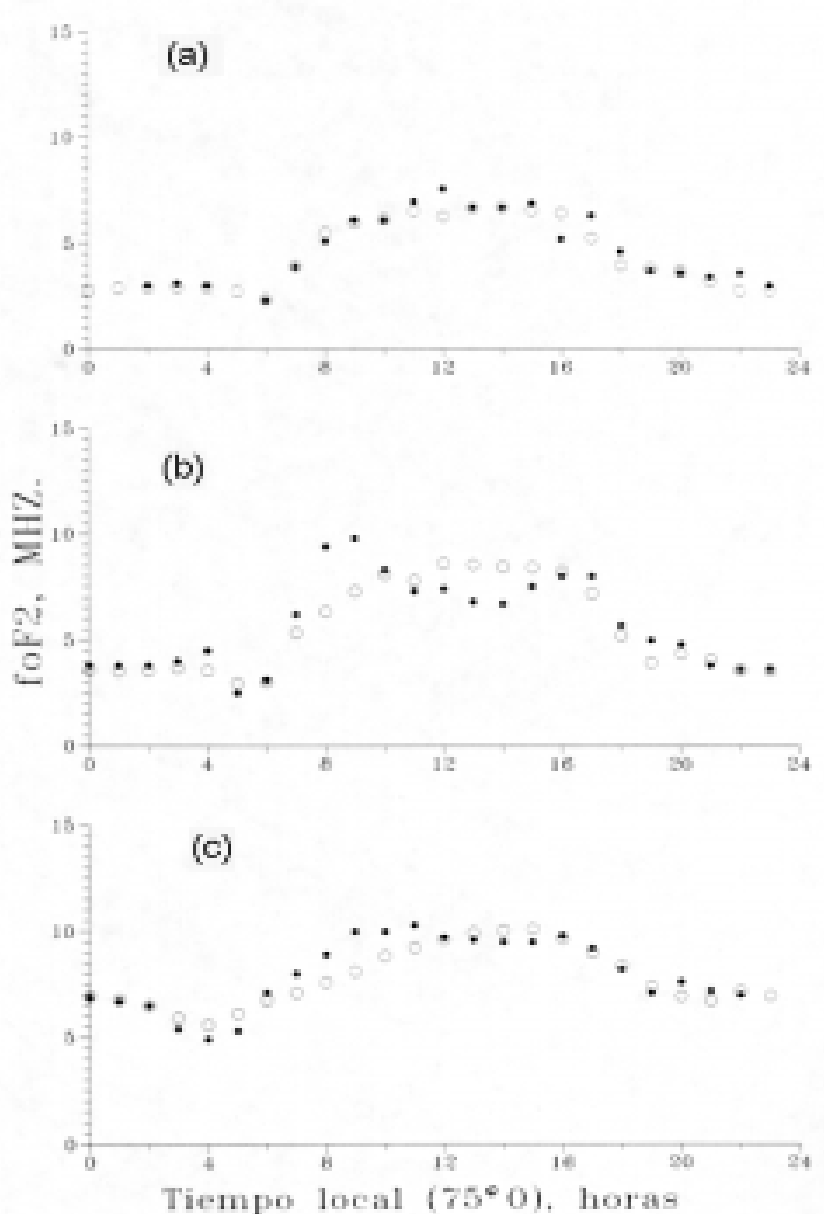

Figura 5 - Comparación entre los valores observados y los valores calculados con el algoritmo propuesto para la frecuencia crítica de la región $\mathrm{F}$ de la ionósfera sobre Concepción $\left(36.8^{\circ} \mathrm{S} ; 73.0^{\circ} \mathrm{O}\right)$. (...) Valores observados. (ooo) Valores calculados. Año 1974. Nivel de actividad geomagnética muy quieto $(0 \mathrm{~d} a p(0.75)<6)$. (a) Invierno (7 de junio). (b) Equinoccio (15 de abril). (c) Verano (30 de noviembre).

Figure 5 - Comparison between observed and calculated values using the proposed algorithm for the ionospheric F-region critical frequency over Concepción $\left(36.8^{\circ} \mathrm{S} ; 73.0^{\circ} \mathrm{W}\right)$. (..) Observed values. (ooo) Calculated values. 1974. Very quiet geomagnetic activity level (0 d ap (0.75) < 6). (a) Winter (7 July). (b) Equinox (15 April). (c) Summer (30 November). en que el cuociente q se elige de la Tab. 1 que lo lista según hora del día, estación del año y el rango de $a p(0.75)$ correspondiente, y foF $2_{\mathrm{dr}}$ es el valor representativo de condiciones geomagnéticas quietas para la hora en cuestión (en la Fig. 4b sólo se muestran los valores de q para invierno). Cabe hacer notar que, aunque para una hora determinada las tablas listan el mismo q para los cuatro meses de una misma estación del año y un nivel de actividad geomagnética fijo, los valores de la foF2 para esos meses no son los mismos porque las correspondientes foF ${ }_{\mathrm{dr}}$, no son iguales (pequeñas diferencias por dependencias con la estación del año y con
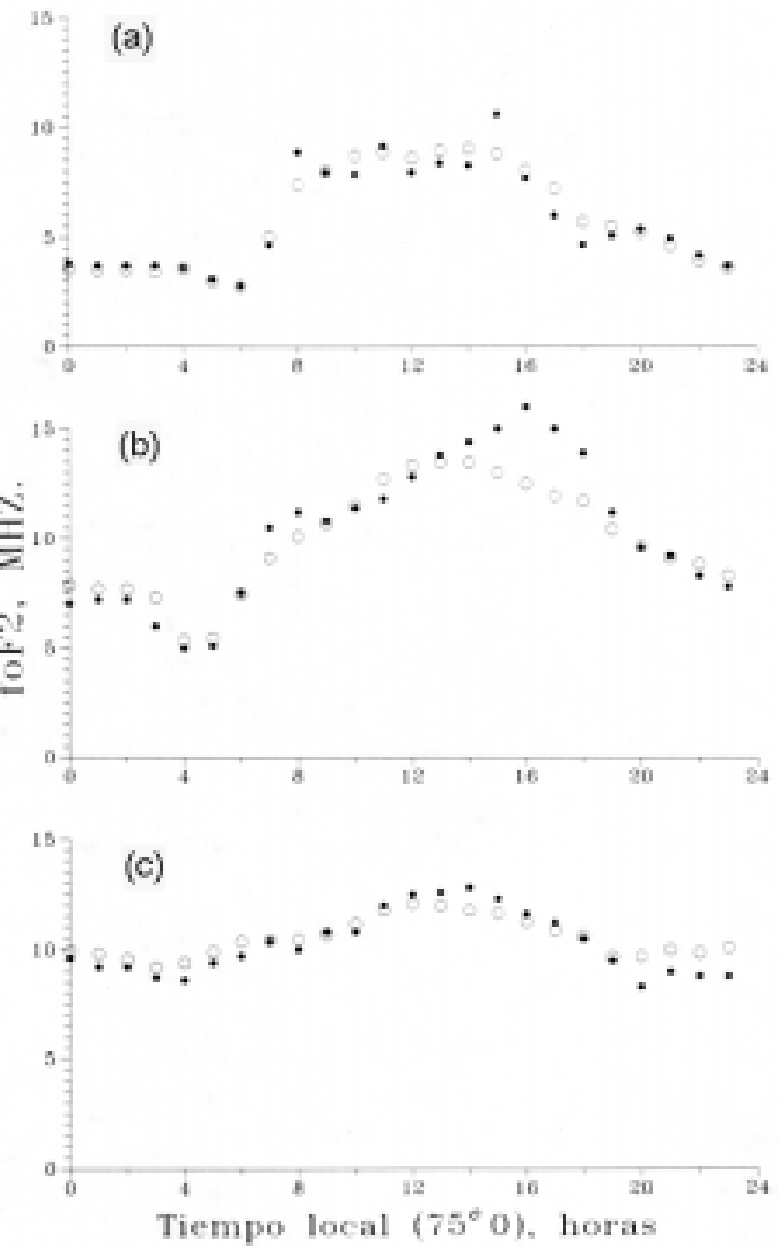

Figura 6 - Comparación entre los valores observados y los valores calculados con el algoritmo propuesto para la frecuencia crítica de la región $\mathrm{F}$ de la ionósfera sobre Concepción $\left(36.8^{\circ} \mathrm{S} ; 73.0^{\circ} \mathrm{O}\right)$. (...) Valores observados. (ooo) Valores calculados. Año 1978. Nivel de actividad geomagnética quieto $(6 \mathrm{~d} a p(0.75)<18)$. (a) Invierno (1 de julio). (b) Equinoccio (1 de octubre). (c) Verano (16 de diciembre).

Figure 6 - Comparison between observed and calculated values using the proposed algorithm for the ionospheric F-region critical frequency over Concepción $\left(36.8^{\circ} \mathrm{S} ; 73.0^{\circ} \mathrm{W}\right)$. (...) Observed values. (ooo) Calculated values. 1978. Quiet geomagnetic activity level (6 d ap $(0.75)<18)$. (a) Winter (1 July). (b) Equinox (1 October). (c) Summer (16 December). 

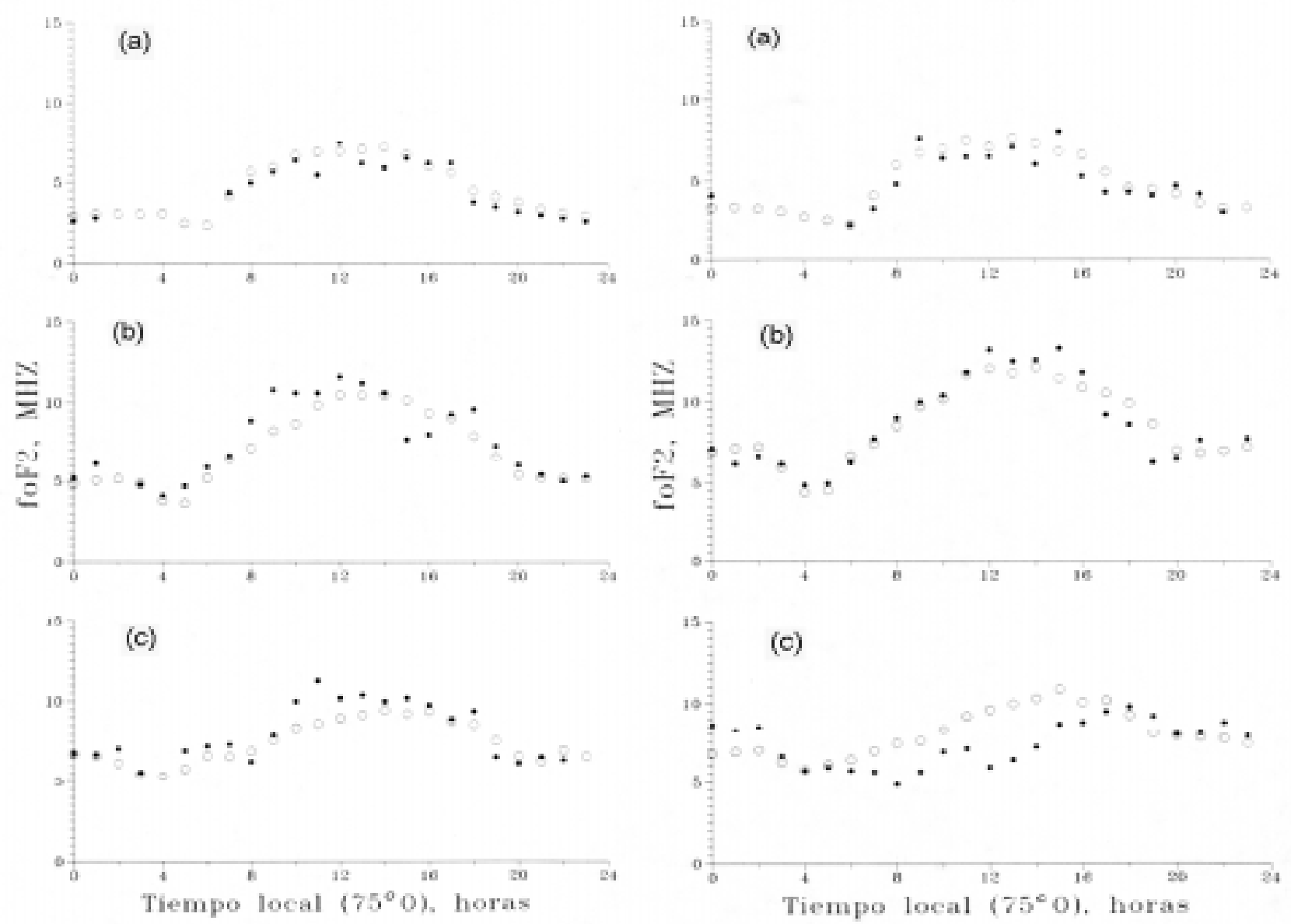

Figura 7 - Comparación entre los valores observados y los valores calculados con el algoritmo propuesto para la frecuencia crítica de la región $\mathrm{F}$ de la ionósfera sobre Concepción $\left(36.8^{\circ} \mathrm{S} ; 73.0^{\circ} \mathrm{O}\right)$. (‥) Valores observados. (ooo) Valores calculados. Año 1974. Nivel de actividad geomagnética perturbado $(18 \mathrm{~d} \operatorname{ap}(0.75)<30)$. (a) Invierno (26 de julio). (b) Equinoccio (1 de octubre). (c) Verano (20 de diciembre).

Figure 7 - Comparison between observed and calculated values using the proposed algorithm for the ionospheric F-region critical frequency over Concepción $\left(36.8^{\circ} \mathrm{S} ; 73.0^{\circ} \mathrm{W}\right)$. ( ..) Observed values. (ooo) Calculated values. 1974. Perturbed geomagnetic activity level (18 d ap $(0.75)<30)$. (a) Winter (26 July). (b) Equinox (1 October). (c) Summer (20 December).

el nivel de actividad solar), y porque los q correspondientes también son en general diferentes (por la presencia o no de actividad magnética episódica a la respectiva hora).

Como se mencionó anteriormente, en contadas ocasiones dentro del intervalo inicial considerado no existen valores de la foF2 que satisfagan simultáneamente las condiciones de actividad magnética quieta y actividad solar muy alta. En estos casos no es posible determinar valores de la foF $2{ }_{\mathrm{mr}}$, por cuanto no es recomendable una extrapolación cuando sólo se tienen valores para los tres niveles de actividad solar mas bajos. En tal situación los valores foF $2_{\mathrm{mr}}$ se determinan mediante un proceso de interpolación, considerando dos procedimientos, según la disponibilidad de datos. El primero consiste en calcular el valor foF 2 mr por interpolación

Figura 8 - Comparación entre los valores observados y los valores calculados con el algoritmo propuesto para la frecuencia crítica de la región $\mathrm{F}$ de la ionósfera sobre Concepción $\left(36.8^{\circ} \mathrm{S} ; 73.0^{\circ}\right.$ O). ( ...) Valores observados. (ooo) Valores calculados. Año 1974. Nivel de actividad geomagnética muy perturbado (30 d ap (0.75)). (a) Invierno (27 de junio). (b) Equinoccio (16 de octubre). (c) Verano (12 de noviembre).

Figure 8 - Comparison between observed and calculated values using the proposed algorithm for the ionospheric $F$-region critical frequency over Concepción $\left(36.8^{\circ} \mathrm{S} ; 73.0^{\circ} \mathrm{W}\right)$. (...) Observed values. (ooo) Calculated values. 1974. Very perturbed geomagnetic activity level (30 d ap(0.75)). (a) Winter (27 June). (b) Equinox (16 October). (c) Summer (12 December).

mediante una función spline cúbica, usando los valores correspondientes a los dos meses consecutivamente anteriores y a los de los dos meses consecutivamente posteriores. El segundo se utiliza cuando también faltan valores foF 2 mr en los meses anteriores o en los posteriores, y consiste en una interpolación con una función spline cúbica con valores de la foF $2_{\text {mr }}$, de meses del mismo nombre al del que falta, dentro del intervalo inicial, cuyos niveles de actividad solar sean similares al del mes faltante.

Todo lo anterior se hizo separadamente también para los intervalos aproximadamente undecenales del 1 de marzo de 1958 al 31 de octubre de 1968 y del 1 de diciembre de 1979 al 30 de junio de 1989. No se hizo el análisis para un intervalo único (1 marzo 1958 a 30 junio 1989) porque la 
dependencia de la foF2 con el nivel de actividad solar, pareciera ser diferente para cada uno de los tres intervalos considerados (Foppiano \& Arriagada, 1993), como lo han sugerido otros autores para otros lugares e intervalos (e.g. Smith \& King, 1981), aun cuando no hay consenso sobre su significancia (Bradley, 1994). La dependencia de la foF2 con el nivel de actividad geomagnética también pareciera diferir de un intervalo a otro.

\section{RESULTADOS}

Haciendo uso del algoritmo propuesto se determinaron los valores de la foF2 para todas las ocasiones en que no había registros, lográndose así una serie completa para el intervalo 1 de Marzo de 1958 al 30 de Junio de 1989. Cabe destacar que en una de esas ocasiones faltaban registros para tres meses completos (septiembre, octubre y noviembre de 1975) por falla de los equipos.

Como no es posible estimar la bondad del algoritmo para ocasiones en que no hay registros, se presentan ejemplos de días representativos de las distintas estaciones año y de los cuatro niveles de actividad geomagnética. Se eligieron aquellos días para los cuales existe el mayor número posible de registros.

En las Figs. 5 al 8 se muestran los valores de la foF2 calculadas con el algoritmo y los valores observados para todos los días seleccionados. En general, hay una buena predicción para los días de invierno en todos los niveles de actividad geomagnética. Lo mismo ocurre para los días de equinoccio y de verano, en condiciones geomagnéticas muy quietas y quietas, aunque en estos casos es posible observar diferencias no despreciables.

La predicción desmejora considerablemente en los días de equinoccio y verano, en condiciones geomagnéticas perturbadas y muy perturbadas, donde para la mayoría de las horas del día se aprecian diferencias significativas.

Probablemente una parte de las diferencias observadas se deba a que no se ha tomado en cuenta que la respuesta de la ionósfera a cambios del nivel de actividad geomagnética puede depender del nivel de actividad solar. Por definición, el cuociente $\mathrm{q}$ es independiente del nivel de actividad solar.

\section{CONCLUSIONES}

Se ha logrado obtener dos series completas de valores de la frecuencia crítica de la región $\mathrm{F}$ sobre Concepción $\left(36.8^{\circ} \mathrm{S} ; 73.0^{\circ} \mathrm{O}\right)$, para el intervalo 1958 - 1989. Una se puede considerar representativa de condiciones geomagnéticas quietas, y por consiguiente puede ser útil para determinar tendencias interdecadales que no incluyan los efectos de la actividad geomagnética. La otra puede ser de valor tanto para el estudio de efectos de carácter episódicos, como para construir una base para la preparación de modelos empíricos.

Se encuentra que, en general, las diferencias entre los valores de la frecuencia crítica calculados con el algoritmo propuesto y los valores observados, cuando estos existen, son mínimas, a excepción de los días de equinoccio y verano en condiciones geomagnéticas perturbadas y muy perturbadas.

\section{AGRADECIMIENTOS}

Se agradece los comentarios recibidos de tres revisores, los que condujeron a una significativa revisión del texto original. Este trabajo ha sido patrocinado por el Fondo Nacional de Desarrollo Científico y Tecnológico (Proyecto FONDECYT N ${ }^{\circ} 1960628$ )

\section{REFERENCIAS}

BRADLEY, P.A. -1994- Further study of foF2 and M(3000) F2 in different solar cycles. Annali di Geofisica, 37:201208 .

FIELD, R.P. \& RISHBETH, H. -1997- The response of the ionospheric F2-layer to geomagnetic activity: an analysis of worldwide data. J. Atmos. Sol. Terr. Phys. 59:163-180

FOPPIANO, A.J. \& ARRIAGADA, M.A. -1993- Efecto del nivel de actividad geomagnética sobre la concentración electrónica máxima de la ionósfera sobre Concepción, para observaciones correspondientes a tres ciclos solares. Tercera Conferencia Latinoamericana de Geofísica Espacial, 1-5 de Noviembre, Habana, Cuba.

RODGER, A.S., WRENN, G.L. \& RISHBETH, H. -1989Geomagnetic storms in the Antarctic F-region. II Physical interpretation. J. Atmos. Terr. Phys. 51:851866.

SMITH, P.A. \& KING, J.W. -1981- Long-Term relationships between sunspots, solar faculae and the ionosphere. J. Atmos. Terr. Phys. 43:1057-1063.

WRENN, G.L. -1987- Time-weighted accumulations ap (t) and $\mathrm{Kp}(\mathrm{t})$. J. Geophys. Res. 92:10125-10129.

WRENN, G.L., RODGER, A.S. \& RISHBETH, H. -1987Geomagnetic storms in the Antarctic F-region I. Diurnal and seasonal patterns for main phase effects. J. Atmos. Terr. Phys. 49:901-913. 


\section{ALGORITHM TO FILL IN IONOSPHERIC F-REGION CRITICAL FREQUENCY SERIES FOR CONCEPCIÓN, CHILE}

A complete series of foF2 hourly values considered representative of geomagnetic quiet conditions is determined using the incomplete series of observed hourly values at Concepción $\left(36.8^{\circ} \mathrm{S} ; 73.0^{\circ} \mathrm{W}\right)$ in the 1 March 1958 - 1 July 1989 interval. This is carried out in four steps, closely following procedures published by Wrenn et al. (1987) and Rodger et al. (1989) as follows. foF2 hourly values, corresponding to low geomagnetic activity (as measured by a weighted geomagnetic activity index), for the 1 November 1968 - 30 November 1979 subinterval are first selected. These are grouped according to time-of-day, month and four solar activity levels (as measured by solar flux in the $10.7 \mathrm{~cm}$ band) independent of year, and monthly mean values are determined (Fig. 1). Then representative monthly values, foF $2_{\mathrm{mr}}$, are determined for each month of each year by interpolation between monthly mean values corresponding to the two nearest solar activity levels using the monthly mean solar activity for the given month (Fig. 1). Assigning these foF $2_{\text {mr }}$, values to the median day of the month, representative hourly values, foF $2_{\mathrm{dr}}$, for each day of the sub-interval are calculated by interpolation (Fig. 2). The complete foF 2 dr hourly series thus obtained for the whole sub-interval takes into account known seasonal and solar activity level dependencies (Fig. 3).

Computing the ratio, $\mathrm{q}$, between observed foF 2 and the corresponding foF 2 dr then permits the dependency of foF2 hourly values on geomagnetic activity to be determined. Values of $\mathrm{q}$ are grouped according to timeof-day, month and four levels of geomagnetic activity (again as measured by a weighted geomagnetic activity index) independent of year, and monthly mean values are determined (Fig. 4a). These are a measure of the foF2 dependency on geomagnetic activity. In practice, $\mathrm{q}$ monthly mean values for months corresponding to the same season are very similar, and thus only seasonally mean values are needed (Fig. 4b and Table 1).

The proposed algorithm to compute the sub-interval foF2 missing values uses the simple expression

$$
\mathrm{foF} 2=\mathrm{q} \text { foF } 2_{\mathrm{dr}}
$$

where values of $\mathrm{q}$ are selected from Table 1 and foF $2_{\mathrm{dr}}$ are those referred to as the complete series of hourly values of foF 2 considered representative of geomagnetic quiet conditions already obtained.

The same procedures indicated above are used independently for the other approximately undecenal subintervals 1 March 1958 - 31 October 1968 and 1 December 1979 - 30 June 1989 (the corresponding q tables are not reproduced here).

Since there is no way to assert the goodness of the proposed algorithm when there is no observed foF2 values, calculated and observed values are compared for a few days for which they do exist. Days considered representative of different seasons and levels of solar and geomagnetic activity are used (Figs. 5 to 8 ).

Summarizing: (a) Two complete series of foF2 hourly values have been obtained spanning 30 years. One can be considered representative of quiet geomagnetic conditions and thus can be useful to deriving long term trends that are independent of geomagnetic activity. The other can be of value both to study event effects and to serve as a base for empirical modelling; (b) Differences between calculated and observed values are not significant except for equinox and summer conditions at high geomagnetic activity level.

\section{NOTAS SOBRE LOS AUTORES}

\section{M.A. Arriagada.}

Profesor de Estado en Física, Universidad de Chile y Magister en Física, Universidad Austral de Chile. Se desempeña actualmente como Profesor Asociado del Departamento de Física de la Universidad del Bío-Bío, Chile. Su área de interés es la Física de la Ionosfera, en particular morfología y dinámica de la región $\mathrm{F}$.

\begin{abstract}
A.J. Foppiano.
Profesor de Estado en Matemáticas y Física, Universidad de Chile y Doctor (Ph.D), Imperial College of Science and Technology, University of London. Se desempeña actualmente como Profesor Titular del Departamento de Física de la Atmósfera y del Océano de la Universidad de Concepción, Chile. Su área de interés es la Física de la Ionosfera, en particular absorción auroral de ondas radioeléctricas y morfología y dinámica de la región $\mathrm{F}$.
\end{abstract}

